\title{
Efficacy of eribulin in women with metastatic breast cancer: a pooled analysis of two phase 3 studies
}

\author{
Chris Twelves $\cdot$ Javier Cortes $\cdot$ Linda Vahdat \\ Martin Olivo $\cdot$ Yi He $\cdot$ Peter A. Kaufman • \\ Ahmad Awada
}

Received: 24 June 2014 / Accepted: 17 September 2014/Published online: 8 November 2014

(c) The Author(s) 2014. This article is published with open access at Springerlink.com

\begin{abstract}
Data from two phase 3 studies of eribulin were pooled in analyses initially requested by the European Medicines Agency to assess whether specific patient subgroups, previously treated with an anthracycline and a taxane, benefited from eribulin. Study 305/EMBRACE included women after two-to-five lines of chemotherapy for advanced breast cancer who were randomized to eribulin mesylate $\left(1.4 \mathrm{mg} / \mathrm{m}^{2}\right.$ on days 1 and 8 every 21 days) or treatment of physician's choice. In Study 301, patients who had received up to two prior chemotherapy regimens for advanced disease were randomized to eribulin (as above) or capecitabine $\left(1.25 \mathrm{~g} / \mathrm{m}^{2}\right.$ b.i.d. on days $1-14$
\end{abstract}

Electronic supplementary material The online version of this article (doi:10.1007/s10549-014-3144-y) contains supplementary material, which is available to authorized users.

\section{Twelves}

Leeds Institute of Cancer and Pathology and St James's Institute of Oncology, Leeds, UK

J. Cortes $(\bowtie)$

Medical Oncology Department, Vall d'Hebron University

Hospital, Paseo Vall d'Hebron 119-129, 08035, Barcelona,

Spain

e-mail: jacortes@vhio.net

L. Vahdat

Weill Cornell Medical College, New York, USA

M. Olivo · Y. He

Eisai Inc., Woodcliff Lake, USA

P. A. Kaufman

Norris Cotton Cancer Center and Dartmouth-Hitchcock Medical Center, Lebanon, USA

A. Awada

Medical Oncology Clinic, Institut Jules Bordet, Université Libre de Bruxelles, Brussels, Belgium every 21 days). In the pooled population, overall survival (OS), progression-free survival and response rates were analysed in the intent-to-treat population and selected subgroups. Overall, 1,062 patients were randomized to eribulin and 802 patients to control. Median OS was 15.2 months with eribulin versus 12.8 months with control (hazard ratio [HR] 0.85; $95 \% \mathrm{CI} 0.77,0.95 ; P=0.003$ ). In all subgroups assessed, OS data favoured eribulin; significant improvements occurred in some subgroups, notably in women with human epidermal growth factor receptor 2 (HER2)-negative disease (HR 0.82; $P=0.002$ ), although the effect in those with HER2-negative but hormone-receptor-positive disease did not reach statistical significance; benefits were also seen, among others, in those with estrogen-receptor-negative and triple-negative disease. Eribulin improves OS in various patient subgroups with advanced/metastatic breast cancer who had previously received an anthracycline and a taxane. Women with HER2-negative disease are among those who may obtain benefit from eribulin.

Keywords Eribulin mesylate $\cdot$ Halaven $\cdot$ Metastatic breast cancer . Pooled analysis

\section{Introduction}

Despite improvements in treatment, long-term survival for women with advanced breast cancer remains poor, with a 5-year survival rate of less than $25 \%$ for those presenting with metastatic disease [1]. Effective treatment options with proven survival benefits are therefore required for these patients. It is, however, often difficult for clinicians to make evidence-based judgements regarding the most effective treatment for such patients. For example, it is 
increasingly common for patients to receive anthracyclineand/or taxane-based regimens in the adjuvant or first-line metastatic settings. A systematic review by Oostendorp et al. in 2011 demonstrated the paucity of good-quality, randomized clinical trial data on chemotherapy for patients with advanced disease who have previously received an anthracycline and a taxane [2]. Available evidence suggests that sequential single agent treatment is generally preferable to combination treatment $[3,4]$. In addition, there is some evidence that re-challenging patients with an anthracycline or a taxane may be beneficial in the metastatic setting [5, 6], particularly when the disease-free interval has been long; the evidence base is, however, limited. This lack of high-quality, evidence-based data has precluded guidelines from giving clear recommendations as to the sequence in which potentially active chemotherapy agents are best used in women with metastatic breast cancer who have received an anthracycline and a taxane [7, 8].

Since publication of the aforementioned systematic review [2], two phase 3, randomized, controlled trials of eribulin in women with advanced/metastatic breast cancer who have previously received an anthracycline and a taxane have been reported. In Study 305/EMBRACE, eribulin was associated with improved overall survival (OS) compared with treatment of physician's choice (TPC) in patients who had received at least two prior chemotherapy regimens for advanced disease, and no more than five cytotoxic regimens that had included an anthracycline and a taxane [9]. These results were confirmed in an updated analysis (based on a $77 \%$ event rate) that was requested by US and European regulatory authorities [9]. In Study 301, which involved patients in an earlier line setting than Study 305/EMBRACE, a statistically significant survival benefit for eribulin over capecitabine was not shown (manuscript in press, J Clin Oncol). In a pre-specified subgroup analysis, however, median OS was longer with eribulin than with capecitabine in patients with human epidermal growth factor receptor 2 (HER2)-negative or triple-negative breast cancer. By contrast, subgroup analyses in Study 305/EMBRACE did not show a significant difference in these populations in either the initial (55\% event rate) or updated analysis (Online Resource 1). A similar hazard ratio (HR) in favour of eribulin was observed in patients with triple-negative breast cancer in the initial analysis of Study 305/EMBRACE and Study 301 (HR 0.71 and 0.70, respectively) [10], but not in the updated analysis of Study 305/EMBRACE. In patients with HER2-positive disease, there was a trend towards a benefit with eribulin in the initial analysis of Study 305/EMBRACE, which became significant in the updated analysis (Online Resource 1); there was no evidence of a benefit in Study 301 (HR 0.97) [10]. The reasons for the differences between the two studies are unclear, but treatment decisions would be aided by greater clarity regarding the effect of eribulin in specific subgroups of women with advanced/metastatic breast cancer.

To provide more information on the efficacy of eribulin in patients with breast cancer with respect to HER2 status, the European Medicines Agency (EMA) requested a pooled analysis of Study 305/EMBRACE and Study 301 by HER 2 and triple-negative disease status. Here, we report the results of this and additional subgroup analyses that provide further insights regarding the effect of eribulin in patients with advanced breast cancer.

\section{Methods}

\section{Study 305/EMBRACE}

Detailed methods for Study 305/EMBRACE have been published previously $[9,10]$. In brief, this was an openlabel, randomized study of eribulin mesylate $\left(1.4 \mathrm{mg} / \mathrm{m}^{2}\right.$ i.v. [equivalent to $1.23 \mathrm{mg} / \mathrm{m}^{2}$ eribulin expressed as free base] on days 1 and 8 every 21 days) versus TPC in women with locally recurrent or metastatic breast cancer. Patients were randomized in a 2:1 ratio to eribulin and TPC, respectively. A key inclusion criterion was that patients had received between two and five previous chemotherapy regimens (including an anthracycline and a taxane), two or more of which were for locally recurrent or metastatic disease. Known brain metastases, unless treated and stable, and pre-existing neuropathy of grade three or higher were among the key exclusion criteria. Study treatment continued until: disease progression; unacceptable toxic effects; the patient or physician requested discontinuation; or serious non-compliance with the protocol. The primary objective was to compare OS between the two treatment arms.

\section{Study 301}

Detailed methods for Study 301 have been presented [11; manuscript in press, J Clin Oncol]. This was an open-label, randomized study of eribulin mesylate $\left(1.4 \mathrm{mg} / \mathrm{m}^{2}\right.$ i.v. on days 1 and 8 every 21 days) versus capecitabine $\left(1.25 \mathrm{~g} / \mathrm{m}^{2}\right.$ b.i.d. on days 1-14 every 21 days; $1: 1$ ratio) as first-, second-, or third-line chemotherapy for advanced breast cancer. Inclusion criteria included: no more than three prior chemotherapy regimens, of which no more than two were for advanced or metastatic disease; and prior therapy with an anthracycline and a taxane. Among the exclusion criteria were prior capecitabine treatment and radiation therapy encompassing more than $30 \%$ of marrow. Similar to Study 305/EMBRACE, patients received study treatment until 
disease progression, unacceptable toxicity, or the patient or physician requested discontinuation of treatment. In contrast to Study 305/EMBRACE, Study 301 had co-primary endpoints of OS and progression-free survival (PFS). The study would be defined positive if, at final analysis, either OS with eribulin was statistically significantly better $(P \leq 0.0372)$ than with capecitabine, or PFS with eribulin was statistically significantly better $(P \leq 0.01)$ than with capecitabine, and HR for OS (eribulin/capecitabine) was less than 1.0.

\section{Ethics}

Both Study 305/EMBRACE and Study 301 required informed patient consent and approval by ethical review boards, and were conducted in line with the World Medical Association revised Declaration of Helsinki and guidelines of the International Conference on Harmonisation of Good Clinical Practice.

Pooled analysis

The objective of this pooled analysis (prompted initially by a request from the EMA for an analysis by HER2 status) was to assess OS in the intent-to-treat (ITT) population and in important subgroups of patients with breast cancer. Data from the updated analysis (based on $77 \%$ event rate) of Study 305/EMBRACE were used in this analysis. The analysis included subgroups based on: HER2 status (positive, negative or unknown); estrogen-receptor (ER) status (positive, negative or unknown); progesterone-receptor status (positive, negative, unknown or data not shown); the number of organs involved (two or fewer, or more than two); presence of visceral disease (yes or no); and taxane resistance (refractory: yes or no). Patients were defined as refractory to taxanes if they experienced progression within 60 days after taking their last taxane dose. Other subgroups were patients with or without triple-negative disease (or unknown) and those who were HER2-negative but hormone-receptor-positive. Response rates and PFS were also assessed for the overall population and the various subgroups. Investigator review data were used for these analyses because the independent review committee data may have been underestimated owing to informative censoring (and were available only after $55 \%$ of events had been reached in Study 305).

In this analysis, adjustment for study was necessary due to the 2:1 randomization in Study 305/EMBRACE. This was to overcome bias that would otherwise be introduced owing to the larger number of eribulin patients receiving treatment in a later treatment-line setting. Thus, median OS and PFS data were derived from survival curves adjusted by study using methodology outlined previously by Chang et al. and Makuch [12, 13]. Cox regression analysis was used to calculate HRs for OS and PFS stratified as per the original studies with the addition of stratification by study. Thus, data were stratified by region (region 1: North America, Western Europe, Australia; region 2: Latin and South America; region 3: Eastern Europe; region 4: Asia), prior capecitabine use and study. Analyses in the overall population were additionally stratified for HER2 status. For analysis of patients with HER2-negative disease, data were stratified for triple-negative disease, in addition to region, prior capecitabine and study. $P$ values for HRs were based on two-sided stratified log-rank tests and may be considered nominal owing to the unplanned nature of the analysis. Interaction analyses were performed for subgroup data using Cox models. Differences in investigatorreviewed response rates were tested by the CochraneMantel-Haenszel test stratified by study.

\section{Results}

Patients

In total, 1,062 patients were randomized to eribulin and 802 patients to control (TPC or capecitabine). Patients in the eribulin and control groups were generally well matched (Table 1). In the eribulin group, patients had most commonly received two prior chemotherapy regimens for advanced disease (35.1\% compared with $29.4 \%$ in the control group), whereas patients had most commonly received one regimen for advanced disease in the control group (37.4\% compared with $27.1 \%$ in the eribulin group). This reflects that patients with different levels of pre-treatment were eligible for the individual studies. Accordingly, more than half of the patients in Study 301 had received only one prior regimen for advanced disease, whereas in Study 305/EMBRACE patients had most commonly received two regimens for advanced disease.

\section{Efficacy}

The survival curve for OS in the total population showed an early separation in favour of eribulin that was maintained throughout the analysis period (Fig. 1). Median OS in the ITT population was 15.2 months in the eribulin arm compared with 12.8 months in the control arm, a difference of 2.4 months; analysis showed a significant benefit in favour of eribulin (HR 0.85; $P=0.003$ ). In addition, treatment with eribulin was associated with apparent benefits in OS across all patient subgroups (Fig. 2). A 2.9month difference in OS was found in patients with HER2negative disease (median OS, eribulin vs control: 15.2 vs 12.3 months, respectively; HR $0.82 ; P=0.002$ ), although this effect did not reach statistical significance in patients 
Table 1 Baseline demographics and characteristics of the total ITT population

\begin{tabular}{lrr}
\hline & $\begin{array}{l}\text { Eribulin } \\
(n=1,062)\end{array}$ & \multicolumn{1}{c}{$\begin{array}{l}\text { Control } \\
(n=802)\end{array}$} \\
\hline $\begin{array}{l}\text { Median age, years (range) } \\
\text { Race, } n(\%)\end{array}$ & $55.0(24-85)$ & $54.0(26-81)$ \\
Black & $35(3.3)$ & $30(3.7)$ \\
White & $966(91.0)$ & $728(90.8)$ \\
Asian/Pacific Islander & $21(2.0)$ & $20(2.5)$ \\
Other & $40(3.8)$ & $24(3.0)$ \\
ECOG performance status, $n(\%)$ & & $333(41.5)$ \\
0 & $467(44.0)$ & $427(53.2)$ \\
1 & $537(50.6)$ & $38(4.7)$ \\
2 & $50(4.7)$ & $1(0.1)$ \\
3 & 0 & \\
Number of prior chemotherapy regimens & & 0 \\
0 & $148(13.9)$ & $153(19.1)$ \\
1 & $384(36.2)$ & $345(43.0)$ \\
2 & $260(24.5)$ & $161(20.1)$ \\
3 & $267(25.1)$ & $142(17.7)$ \\
$\geq 4$ &
\end{tabular}

Number of prior chemotherapy regimens for advanced disease

$\begin{array}{lrr}0 & 117(11.0) & 104(13.0) \\ 1 & 288(27.1) & 300(37.4) \\ 2 & 373(35.1) & 236(29.4) \\ >2 & 284(26.7) & 161(20.1) \\ \text { Number of organs involved, } n(\%) & & \\ 1 & 198(18.6) & 127(15.8) \\ 2 & 346(32.6) & 259(32.3) \\ 3 & 298(28.1) & 226(28.2) \\ \geq 4 & 218(20.5) & 189(23.6) \\ \text { Site of disease, } n(\%) & & \\ \text { Visceral } & 880(82.9) & 694(86.5) \\ \text { Non-visceral only } & 171(16.1) & 101(12.6) \\ \text { Missing } & 11(1.0) & 7(0.9) \\ \text { HER2 status, } n(\%) & & 123(15.3) \\ \text { Positive } & 169(15.9) & 572(71.3) \\ \text { Negative } & 748(70.4) & 107(13.3) \\ \text { Unknown } & 145(13.7) & \\ \text { ER status, } n(\%) & & 449(56.0) \\ \text { Positive } & & 288(35.9) \\ \text { Negative } & 595(56.0) & 65(8.1) \\ \text { Unknown } & & \\ \text { Triple-negative disease, } n(\%) & 243(22.9) & (501(50.1) \\ \text { Taxane refractory, } n(\%)^{\mathrm{a}} & & \\ \text { Yes } & 530(49.9) & (50.0) \\ \text { No } & 532(50.1) & \\ \end{array}$

ECOG Eastern Cooperative Oncology Group, ER estrogen receptor, HER2 human epidermal growth factor receptor 2, ITT intent-to-treat, $P R$ progesterone receptor

${ }^{a}$ Refractory defined as progressed within 60 days after taking the last dose with HER2-negative but hormone-receptor-positive disease $(P=0.060)$. The difference in OS for those with HER2positive disease favoured eribulin but did not reach statistical significance (13.5 vs 12.2 months; HR 0.82; $P=0.135)$; the patient numbers were, however, smaller in this subgroup than in the HER2-negative group (HER2positive, eribulin $n=169$, control $n=123$; HER2-negative, eribulin $n=748$, control $n=572$ ). In patients with triple-negative disease, median survival was 4.7 months longer in patients treated with eribulin than in those who received control (median OS: 12.9 vs 8.2 months; HR 0.74 ; $P=0.006$ ). In addition, median OS was longer with eribulin than with control, regardless of ER status (ERpositive: median OS 16.1 vs 14.3 months, HR 0.86 , $P=0.038$; ER-negative: median OS 12.9 vs 10.1 months, HR $0.79, P=0.007)$.

Considering other subgroups, not based on receptor status, a benefit in favour of eribulin over control treatment was found in patients with more than two organs involved (median OS 13.1 vs 10.5 months; HR 0.77; $P<0.001$ ). Patient subgroups based on the presence or absence of visceral disease also had, on average, longer survival times if patients were treated with eribulin than if they received control (visceral disease: median OS 14.3 vs 12.2 months, HR $0.89, P=0.037$; non-visceral disease: median OS 18.8 vs 16.6 months, HR $0.72, P=0.045$ ). A 3.0 month difference in survival in favour of eribulin occurred in patients not refractory to taxane treatment (median OS 17.4 vs 14.4 months; HR $0.81 ; P=0.007)$; the difference in OS did not reach significance in patients who were refractory to taxanes (median OS 12.8 vs 11.3 months; HR 0.91; $P=0.206)$.

Interaction analysis showed clearest evidence of a greater benefit for eribulin in the case of patients with a higher burden of disease, as indicated by more than two organs involved $(P=0.023$ vs those with two or fewer organs involved). Interaction $P$ values were relatively low (compared with interaction data in most of the other tested subgroups, see Fig. 2) in patients with triple-negative disease $(P=0.154$ vs patients without triple-negative breast cancer) and those with ER-negative disease $(P=0.159$ vs other patients).

As in the individual analyses of Study 305/EMBRACE and Study 301, eribulin appeared to have a greater impact on OS than did PFS. Benefits for eribulin in terms of PFS were, however, apparent in the overall population, patients with HER2-negative and triple-negative disease, those who were ER-positive and those with more than two organs involved (Table 2). The magnitudes of these differences were small and interaction analysis did not indicate that the benefits were specific to these subgroups. 


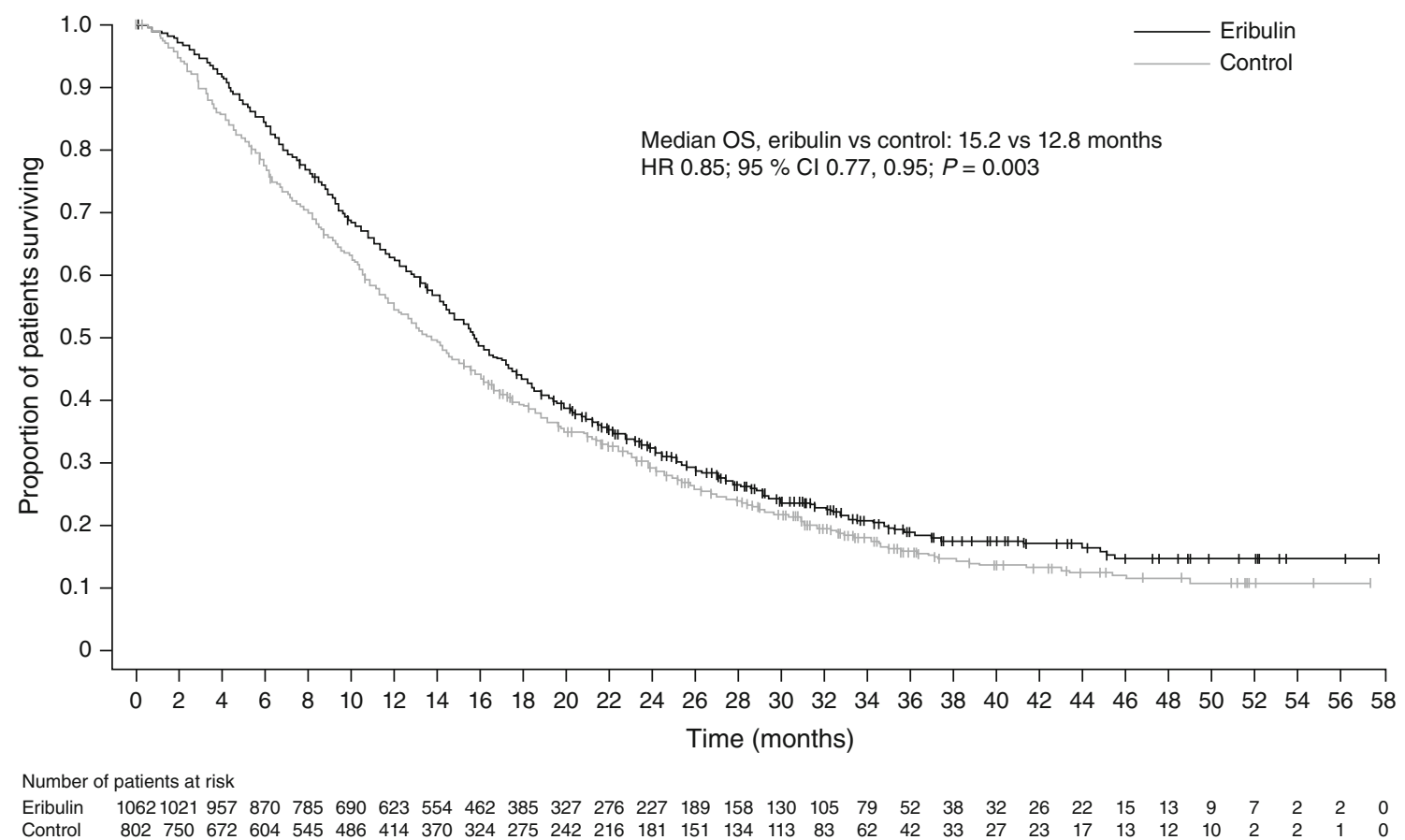

Fig. 1 OS curve in the total intent-to-treat population stratified by study. Survival curve adjusted by study

There were few differences between the treatment groups in objective response rates (ORRs) or clinical benefit rates (CBRs) in the ITT population and in the various subgroups (Online Resource 2). In the ITT population, the ORRs were $14.9 \%$ and $16.4 \%$, and the CBRs were $30.9 \%$ and $30.3 \%$, in the eribulin and control groups, respectively; these differences were not statistically significant. The only significant differences were for CBRs in favour of eribulin in patients with ER-positive disease (33.4\% vs $29.3 \%, P=0.046)$ and those with HER2negative but hormone-receptor-positive disease (33.6\% vs $28.3 \%, P=0.036)$.

\section{Safety}

Tolerability and safety data for Study 305/EMBRACE and Study 301 have been detailed elsewhere $[9,10]$. When the data were pooled, no notable differences in adverse events from those reported in the primary publications were revealed. In the pooled analysis, serious adverse events occurred in similar proportions in the two groups (eribulin $21.1 \%$; control $22.6 \%$ ). In total, $10.5 \%$ of patients in the eribulin group had an adverse event that led to discontinuation, compared with $12 \%$ in the control group.

\section{Discussion}

This pooled analysis showed that eribulin was associated with improved OS in the total population compared with control; it also suggested that certain patients with advanced/metastatic breast cancer may gain a particular benefit from eribulin.

Significant differences in median OS in favour of eribulin over control treatment were seen in most of the subgroups assessed. Women with HER2-negative disease gained a significant survival benefit from eribulin, and a benefit was also found regardless of triple-negative status (a lower HR was found in patients with triple-negative disease). This was also true of patients with ER-positive or ER-negative disease, although the HR indicating a benefit for eribulin was more favourable in the ER-negative subgroup than in the ER-positive subgroup. Generally, similar results were found in an analysis of patients who had previously received one or more lines of chemotherapy for advanced disease (Online Resource 3). A numerical difference in OS in favour of eribulin was seen in patients with HER2-positive breast cancer, and the HR was very similar to that observed in women with HER2-negative disease. The difference between treatments in the HER2positive group was not statistically significant; patient numbers were, however, substantially smaller in this subgroup, resulting in a wide confidence interval.

Considering other patient subgroups, those with more than two affected organs gained a clear additional survival benefit from eribulin treatment compared with control. In one report, the response to chemotherapy tends to decrease as the number of organs involved increases [14]. Improved response rates in patients with multiple organs involved have, however, been reported previously; a study of 


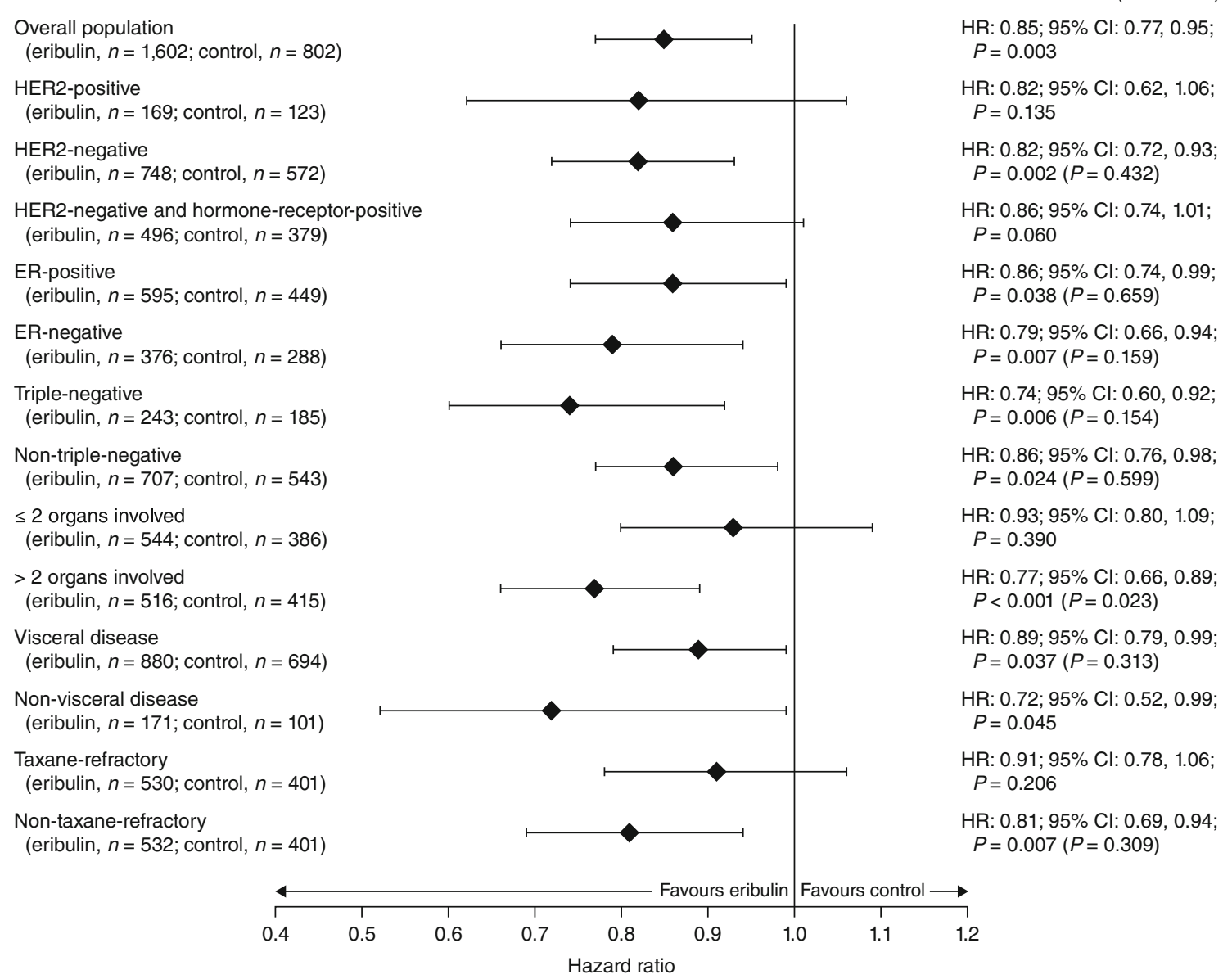

Fig. 2 HRs for OS in the overall population and subgroups based on HER2 status, presence of triple-negative disease, number of organs involved, presence of visceral disease and resistance to taxane treatment. HR based on Cox proportional hazards stratified by geographic region, prior capecitabine use and study (overall

docetaxel in patients with advanced breast cancer reported the highest response rate in those with more than two organs involved $(83.3 \%$, compared with $62.5 \%$ in those with one organ involved and $45.5 \%$ in those with two organs involved) [15]. The reasons for the results observed in the present study are unclear. Patients with multiple organs involved are more likely to have triple-negative disease [16-18]. It could be, therefore, that the apparent survival benefits with eribulin observed among patients with more than two organs involved were influenced by a high proportion of patients with triple-negative breast cancer in that subgroup; this observation may, alternatively, be due to chance alone. This finding is, moreover, of limited clinical relevance, because the number of organs involved does not typically impact on treatment selection.

Patients also benefited from eribulin regardless of whether they had visceral or non-visceral disease. In addition, those who were not refractory to taxanes had improved OS if treated with eribulin compared with population additionally stratified by HER2 status; HER2-negative population additionally stratified by triple-negative status). $P$ value based on stratified log-rank test. Interaction $P$ values (Cox model) are given for subgroups for which significant differences were apparent

control. As expected, the current analysis did not reveal any notable differences in safety compared with the previously reported data.

Interaction analyses, which were not part of the initial pooled analysis plan, showed a distinct additional benefit of eribulin over control treatment for patients with more than two organs involved compared with other patients. In addition, relatively low interaction $P$ values in the ERnegative and triple-negative subgroups suggested there may be an enhanced benefit from eribulin in these groups.

Statistically significant differences in PFS in favour of eribulin over control were found in the ITT population and in some of the subgroups in which significant differences in OS were found. The magnitudes of the differences were, however, small and not clinically significant. Why eribulin may be associated with more meaningful differences in OS than in PFS is not clearly understood.

Given the lack of high-quality studies in patients with advanced breast cancer previously treated with taxanes and 
Table 2 PFS in the ITT population and selected subgroups

$E R$ estrogen receptor, $C I$ confidence interval, HER2 human epidermal growth factor receptor 2, $H R$ hazard ratio, ITT intent-to-treat, $P F S$ progressionfree survival, $P R$ progesterone receptor

${ }^{a}$ Based on curve adjusted by study

${ }^{\mathrm{b}}$ Cox proportional hazards stratified by geographic region, prior capecitabine use and study (overall population additionally stratified by HER2 status; HER2-negative population additionally stratified by triplenegative status)

c Based on stratified log-rank test

${ }^{\mathrm{d}}$ Interaction $P$ values (Cox model) are given for subgroups for which significant differences were apparent

\begin{tabular}{|c|c|c|c|c|c|}
\hline & \multicolumn{2}{|c|}{ Median PFS, months ${ }^{\mathrm{a}}$} & \multirow[t]{2}{*}{$\operatorname{HR}(95 \% \mathrm{CI})^{\mathrm{b}}$} & \multirow[t]{2}{*}{$P$ value $^{\mathrm{c}}$} & \multirow{2}{*}{$\begin{array}{l}P \text { value } \\
\text { (interaction) }\end{array}$} \\
\hline & Eribulin & Control & & & \\
\hline Overall population & 4.0 & 3.4 & $0.90(0.81,0.997)$ & 0.046 & \\
\hline \multicolumn{6}{|l|}{ HER2 status } \\
\hline Positive & 4.0 & 4.7 & $1.02(0.78,1.34)$ & 0.865 & 0.185 \\
\hline Negative & 3.7 & 3.0 & $0.84(0.74,0.95)$ & 0.006 & \\
\hline $\begin{array}{l}\text { HER2 negative/ } \\
\text { hormone receptor positive }\end{array}$ & 4.2 & 3.4 & $0.87(0.75,1.02)$ & 0.084 & \\
\hline \multicolumn{6}{|l|}{ ER status } \\
\hline Positive & 4.2 & 3.7 & $0.86(0.74,0.99)$ & 0.040 & 0.527 \\
\hline Negative & 3.0 & 2.9 & $0.89(0.75,1.06)$ & 0.212 & \\
\hline Triple negative & 2.8 & 2.6 & $0.78(0.63,0.96)$ & 0.018 & 0.117 \\
\hline Non-triple negative & 4.2 & 3.8 & $0.926(0.82,1.04)$ & 0.21 & \\
\hline \multicolumn{6}{|l|}{ Number of organs involved } \\
\hline$\leq 2$ & 4.2 & 4.2 & $0.92(0.79,1.07)$ & 0.290 & 0.468 \\
\hline$>2$ & 3.7 & 2.8 & $0.86(0.74,0.99)$ & 0.043 & \\
\hline \multicolumn{6}{|l|}{ Visceral disease } \\
\hline Yes & 3.8 & 3.3 & $0.94(0.84,1.05)$ & 0.274 & \\
\hline No & 4.7 & 4.3 & $0.75(0.56,1.02)$ & 0.065 & \\
\hline \multicolumn{6}{|l|}{ Taxane refractory } \\
\hline Yes & 3.5 & 2.8 & $0.90(0.77,1.05)$ & 0.176 & \\
\hline No & 4.3 & 4.0 & $0.87(0.75,1.01)$ & 0.079 & \\
\hline
\end{tabular}

anthracyclines, the data presented here add to the evidence base for clinicians. The main strength of the current analysis is that the data are derived from well-designed, randomized studies providing high-quality evidence. Pooling the data from these controlled studies has provided further insights into subgroups that may gain a particular advantage from eribulin treatment. Specifically, the individual studies were not powered to demonstrate differences in patient subgroups. This is especially relevant in the context of HER2 status. Both Study 305/EMBRACE and Study 301 included patients with either HER2-positive or HER2negative disease. This reflected trial design and clinical practice at a time when HER2-targeted therapy was not routinely continued in combination with successive chemotherapy regimens and there were not multiple agents targeting the HER family. The pooled analysis gave us sufficient numbers of patients to look at outcomes by HERand ER-receptor status.

There are, however, limitations to the current analysis. First, this was an unplanned analysis, albeit one undertaken initially at the request of a regulatory body. Pooling the data was complicated by the fact the two studies involved different patient groups, in particular with regard to the extent of prior chemotherapy. The analytical plan did, however, take into account this difference to some extent. Treatment in the control arms was also not the same, with Study 305/EMBRACE using TPC and Study 301 using capecitabine. Nevertheless, the subgroup data presented here provide useful insights into specific subgroups of patients with advanced breast cancer who may gain a greater benefit in terms of survival than others from eribulin treatment.

Patients with HER2-negative disease represent about $85 \%$ of women with metastatic breast cancer [19], and so confirmation from the pooled analysis that these women benefit significantly from eribulin is of particular clinical relevance. The fact that women with triple-negative breast cancer may derive a particularly strong benefit from eribulin is worthy of further investigation. Interestingly, in vitro and in vivo data suggest that eribulin treatment may promote transition of triple-negative breast cancer cells from a mesenchymal to an epithelial phenotype, and that this is coupled with a decrease in the ability of these cells to migrate and therefore metastasize [20]. The added OS benefit of eribulin in women with HER2-positive disease remains unclear because it did not reach statistical significance. As noted previously, patients with HER2positive disease in Study 305/EMBRACE experienced a significant benefit with eribulin in the updated analysis, whereas there was no such benefit in Study 301. There is no clear explanation for this difference between the studies; the impact of post-progression therapies is being investigated. The question of the single agent activity of eribulin in women with HER2-positive metastatic breast cancer is, 
however, of less relevance clinically now that combination with HER2-targeted therapy is acknowledged to be optimal across multiple lines of therapy. Nevertheless, our data suggest that eribulin is active as a single agent in women with HER2-positive disease, and preliminary results in combination with trastuzumab are encouraging [21].

\section{Conclusion}

This pooled analysis of women with advanced/metastatic breast cancer confirms the significant survival benefit of eribulin compared with control following treatment with a taxane and an anthracycline in either the adjuvant or the metastatic setting. This survival benefit holds true across most patient subgroups. Indeed, women with HER2-negative or triple-negative disease gained a particular benefit; the effects in patients with HER2-negative but hormonereceptor-positive disease were somewhat less robust. Similar findings occurred in the subgroup of patients receiving eribulin in a second-line or later setting.

Acknowledgments The authors wish to thank all the Study 305/EMBRACE and Study 301 investigators, and Jantien Wanders for input into both studies. The authors also thank Andrew Sheridan from Oxford PharmaGenesis ${ }^{\mathrm{TM}}$ Ltd who provided editorial support through funding by Eisai. EMBRACE and the eribulin Study 301 were funded by Eisai.

Conflict of interest CT has received consultancy fees from AstraZeneca, Eisai and Pfizer, and honoraria for presenting from Cephalon, Eisai and Roche. LV has received consultancy fees and honoraria from Eisai. JC has received consultancy fees from Celgene, Novartis and Roche, and honoraria from Celgene, Eisai, Novartis and Roche. PK reports consultancy fees, research funding and honoraria from Eisai. MO and YH are employees of Eisai.

Open Access This article is distributed under the terms of the Creative Commons Attribution Noncommercial License which permits any noncommercial use, distribution, and reproduction in any medium, provided the original author(s) and the source are credited.

\section{References}

1. National Cancer Institute (2013) SEER Cancer Statistics Review, 1975-2010. National Cancer Institute, Bethesda, based on November 2012 SEER data submission, posted to the SEER web site. http://seer.cancer.gov/csr/1975_2010. Accessed 18 Jul 2013

2. Oostendorp LJ, Stalmeier PF, Donders AR, van der Graaf WT, Ottevanger PB (2011) Efficacy and safety of palliative chemotherapy for patients with advanced breast cancer pretreated with anthracyclines and taxanes: a systematic review. Lancet Oncol 12:1053-1061. doi:10.1016/S1470-2045(11)70045-6

3. Dear RF, McGeechan K, Jenkins MC, Barratt A, Tattersall MH, Wilcken N (2013) Combination versus sequential single agent chemotherapy for metastatic breast cancer. Cochrane Database Syst Rev 12:CD008792. doi:10.1002/14651858.CD008792.pub2
4. Andreopoulou E, Sparano JA (2013) Chemotherapy in patients with anthracycline- and taxane-pretreated metastatic breast cancer: an overview. Curr Breast Cancer Rep 5:42-50. doi:10.1007/ s12609-012-0097-1

5. Al-Batran SE, Guntner M, Pauligk C, Scholz M, Chen R, Beiss B, Stopatschinskaja S, Lerbs W, Harbeck N, Jager E (2010) Anthracycline rechallenge using pegylated liposomal doxorubicin in patients with metastatic breast cancer: a pooled analysis using individual data from four prospective trials. $\mathrm{Br} \mathrm{J}$ Cancer 103:1518-1523. doi:10.1038/sj.bjc.66059616605961

6. Toulmonde M, Madranges N, Brouste V, Donamaria C, MacGrogan G, Durand M, Bonnefoi H, Mauriac L, Debled M (2012) Docetaxel rechallenge after a first response in non-resistant metastatic breast cancer: significant activity with manageable toxicity. Breast Cancer Res Treat 134:325-332. doi:10.1007/ s10549-012-2060-2

7. National Comprehensive Cancer Network (2013) NCCN clinical practice guidelines in oncology breast cancer, version 3. http:// www.nccn.org/professionals/physician_gls/PDF/breast.pdf. Accessed 18 Jul 2013

8. Cardoso F, Harbeck N, Fallowfield L, Kyriakides S, Senkus E (2012) Locally recurrent or metastatic breast cancer: ESMO clinical practice guidelines for diagnosis, treatment and followup. Ann Oncol 23:vii11-vii19. doi:10.1093/annonc/mds232

9. Cortes J, O'Shaughnessy J, Loesch D, Blum JL, Vahdat LT, Petrakova K, Chollet P, Manikas A, Dieras V, Delozier T, Vladimirov V, Cardoso F, Koh H, Bougnoux P, Dutcus CE, Seegobin S, Mir D, Meneses N, Wanders J, Twelves C (2011) Eribulin monotherapy versus treatment of physician's choice in patients with metastatic breast cancer (EMBRACE): a phase 3 open-label randomised study. Lancet 377:914-923. doi:10.1016/S01406736(11)60070-6

10. Twelves C, Akerele C, Wanders J, Cortes JA (2010) Eribulin mesylate (E7389) vs treatment of physician's choice (TPC) in patients (pts) with metastatic breast cancer (MBC): subgroup analyses from the EMBRACE study. Ann Oncol 21(Suppl 8):Abstract 2750

11. Kaufman PA, Awada A, Twelves C, Yelle L, Perez EA, Wanders J, Olivo MS, He Y, Dutcus CE (2012) A phase III, open-label, randomized, multicenter study of eribulin mesylate versus capecitabine in patients with locally advanced or metastatic breast cancer previously treated with anthracyclines and taxanes. Cancer Res 72(24 Suppl):Abstract S6-6. doi:10.1158/0008-5472. SABCS12-S6-6

12. Chang IM, Gelman R, Pagano M (1982) Corrected group prognostic curves and summary statistics. J Chronic Dis 35:669-674

13. Makuch RW (1982) Adjusted survival curve estimation using covariates. J Chronic Dis 35:437-443

14. Rahman ZU, Frye DK, Smith TL, Asmar L, Theriault RL, Buzdar AU, Hortobagyi GN (1999) Results and long term follow-up for 1581 patients with metastatic breast carcinoma treated with standard dose doxorubicin-containing chemotherapy: a reference. Cancer 85:104-111

15. Fumoleau P, Chevallier B, Kerbrat P, Krakowski Y, Misset JL, Maugard-Louboutin C, Dieras V, Azli N, Bougon N, Riva A, Roche H (1996) A multicentre phase II study of the efficacy and safety of docetaxel as first-line treatment of advanced breast cancer: report of the Clinical Screening Group of the EORTC. Ann Oncol 7:165-171

16. Gluz O, Liedtke C, Gottschalk N, Pusztai L, Nitz U, Harbeck N (2009) Triple-negative breast cancer-current status and future directions. Ann Oncol 20:1913-1927. doi:10.1093/annonc/mdp492

17. Dent R, Trudeau M, Pritchard KI, Hanna WM, Kahn HK, Sawka CA, Lickley LA, Rawlinson E, Sun P, Narod SA (2007) Triple-negative breast cancer: clinical features and patterns of recurrence. Clin Cancer Res 13:4429-4434. doi:10.1158/1078-0432.CCR-06-3045 
18. Anders CK, Carey LA (2009) Biology, metastatic patterns, and treatment of patients with triple-negative breast cancer. Clin Breast Cancer 9:S73-S81. doi:10.3816/CBC.2009.s.008

19. Howlader N, Altekruse SF, Li CI, Chen VW, Clarke CA, Ries LA, Cronin KA (2014) US incidence of breast cancer subtypes defined by joint hormone receptor and HER2 status. J Natl Cancer Inst 106. doi:10.1093/jnci/dju055

20. Yoshida T, Ozawa Y, Kimura T, Sato Y, Kuznetsov G, Xu S, Uesugi M, Agoulnik S, Taylor N, Funahashi Y, Matsui J (2014)
Eribulin mesilate suppresses experimental metastasis of breast cancer cells by reversing phenotype from epithelial-mesenchymal transition (EMT) to mesenchymal-epithelial transition (MET) states. Br J Cancer 110:1497-1505. doi:10.1038/bjc.2014.80

21. Vahdat L, Schwartzberg L, Wilks S, Rege J, Liao J, Cox D, O'Shaughnessy J (2012) Eribulin mesylate+trastuzumab as firstline therapy for locally recurrent or metastatic HER2-positive breast cancer: results from a phase 2, multicenter, single-arm study. Cancer Res 72(24 Suppl):Abstract P5-20-04 